\title{
Tracheal intubation in an unanticipated difficult airway by advancing a bronchoscope and a tracheal tube introducer through a LMA Supreme ${ }^{\text {TM }}$
}

\author{
Qin-Jun Chu, MD $\cdot$ Xue-Ping Han, MD • \\ Hannah Y. Mak, MSc • David T. Wong, MD
}

Received: 8 August 2011/Accepted: 3 November 2011/Published online: 11 November 2011

(C) Canadian Anesthesiologists' Society 2011

\section{To the Editor,}

We report a case involving a patient with an unanticipated difficult airway in whom tracheal intubation was achieved uneventfully by advancing a bronchoscope and a tracheal tube introducer through a LMA Supreme ${ }^{\mathrm{TM}}$. The patient's written consent was obtained for this publication.

A $70 \mathrm{~kg}$, 52-yr-old woman was scheduled for nephrolithotomy. She had an adequate mouth opening, a normal thyromental distance, and a Mallampati class 3 airway. Anesthesia was induced with midazolam, fentanyl, propofol, and atracurium $i v$. Adequate mask ventilation was achieved. Direct laryngoscopy with a Macintosh \#3 blade showed a Cormack-Lehane Grade 3 laryngeal view and tracheal intubation failed in two attempts. After further mask ventilation, oral bronchoscopic $(5.0 \mathrm{~mm}$ outer diameter [OD]) intubation was attempted but failed due to poor visualization from blood and secretions. The $\mathrm{S}_{\mathrm{P}} \mathrm{O}_{2}$ decreased to $90 \%$. A size 4 LMA Supreme ${ }^{\mathrm{TM}}$ (LMA $^{\mathrm{TM}}$ International, Singapore) was inserted with a satisfactory capnographic waveform and $\mathrm{S}_{\mathrm{P}} \mathrm{O}_{2}$ returned to $100 \%$. A decision was then made to intubate the patient's trachea via the LMA Supreme ${ }^{\mathrm{TM}}$. A pediatric bronchoscope (Olympus BF XP60, $2.8 \mathrm{~mm}$ OD) operated by an anesthesiologist and a solid tracheal tube introducer with a coude tip $(5 \mathrm{~mm}$ OD, Well Lead Medical, China) operated by a second anesthesiologist were advanced through the airway lumen of the LMA Supreme ${ }^{\mathrm{TM}}$ into the pharynx (Figure).

Q.-J. Chu, MD - X.-P. Han, MD

First Affiliated Hospital, Zhengzhou University, Zhengzhou,

China

H. Y. Mak, MSc · D. T. Wong, MD (ه)

Toronto Western Hospital, University Health Network,

University of Toronto, Toronto, ON, Canada

e-mail: david.wong@uhn.ca
The tracheal tube introducer was advanced under bronchoscopic guidance through the glottis into the trachea and positioned $3 \mathrm{~cm}$ above the carina. The bronchoscope and LMA Supreme ${ }^{\mathrm{TM}}$ were then removed while keeping the tracheal tube introducer in position. A $7.0 \mathrm{~mm}$ internal diameter (ID) tracheal tube was railroaded over the tracheal tube introducer and into the trachea, and positioning of the tracheal tube was confirmed by capnography. Ten minutes after the completion of surgery, the patient was awakened and the tracheal tube was removed. The patient's recovery was uneventful.

After failed attempts at tracheal intubation, a supraglottic airway may be inserted for lung ventilation while awaiting alternative airway equipment. In lieu of removing the supraglottic airway to attempt alternative intubation techniques, it can be kept in situ as a conduit for tracheal intubation. ${ }^{1}$ Ventilation is not interrupted and secretions and blood are kept posterior to the supraglottic airway allowing a clear path to the larynx.

The LMA Supreme ${ }^{\mathrm{TM}}$ is a single-use variant of the LMA Proseal ${ }^{\mathrm{TM}}$. Its curved shaft consists of a double lumen, i.e., a central lumen for gastric access within an airway lumen for respiratory tract access. Like the LMA Classic $^{\mathrm{TM}}$, it is difficult to pass an adequately sized endotracheal tube (ETT) directly through the LMA Supreme ${ }^{\mathrm{TM}}$ due to the airway luminal diameter. There are four techniques to achieve tracheal intubation with an adequately sized ETT, through the use of introducers or catheters, via the LMA Supreme ${ }^{\mathrm{TM}}$. The four techniques utilize a small ETT, an Aintree Intubating Catheter, a guidewire, or a tracheal tube introducer. With the first technique, ${ }^{2}$ a small ETT (up to $6.0 \mathrm{~mm}$ ID) is mounted over a bronchoscope and inserted into the trachea through the LMA Supreme ${ }^{\mathrm{TM}}$. Subsequently, an airway exchange catheter is inserted via the ETT, which is later exchanged for a full-sized ETT. 


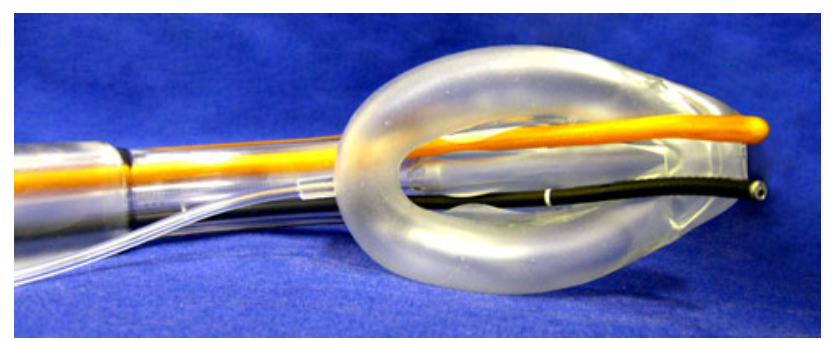

Figure A bronchoscope and a tracheal tube introducer are inserted through the LMA Supreme ${ }^{\mathrm{TM}}$

In the second technique, ${ }^{3}$ an Aintree Intubating Catheter (4.7 $\mathrm{mm} \mathrm{ID)} \mathrm{is} \mathrm{mounted} \mathrm{over} \mathrm{a} \mathrm{bronchoscope} \mathrm{and} \mathrm{inserted}$ through the LMA Supreme ${ }^{\mathrm{TM}}$ into the trachea. The bronchoscope and LMA Supreme ${ }^{\mathrm{TM}}$ are removed and an ETT is railroaded over the catheter.

A third technique ${ }^{4}$ uses a guidewire inserted through the flexible bronchoscope. They are inserted together through the LMA Supreme ${ }^{\mathrm{TM}}$, and then the bronchoscope and LMA Supreme ${ }^{\mathrm{TM}}$ are removed. The guidewire is then loaded with an exchange catheter and railroaded with an ETT. In the fourth technique ${ }^{5}$ (described in patients with normal airways), a tracheal tube introducer, guided by a bronchoscope, is inserted through the LMA Supreme ${ }^{\mathrm{TM}}$. After removal of the bronchoscope and LMA Supreme ${ }^{\mathrm{TM}}$, an ETT is railroaded into position.

In our case, after failed intubation attempts, we inserted the LMA Supreme ${ }^{\mathrm{TM}}$ due to its immediate availability. A LMA Classic ${ }^{\mathrm{TM}}$ or a LMA Proseal ${ }^{\mathrm{TM}}$ could also be used for the same purpose. A LMA Fastrach ${ }^{\mathrm{TM}}$, specifically designed as a conduit for intubation without the use of catheters, could also be used; but it was not available. As for the choice of introducer/catheters, an Aintree Intubating Catheter, other airway exchange catheters, or a guidewire are alternative options, but the tracheal tube introducer was selected due to its immediate availability in every operating room.

In conclusion, we describe management of a failed airway, achieved with assistance from a flexible bronchoscope to guide the insertion of a tracheal tube introducer into a LMA Supreme ${ }^{\mathrm{TM}}$. This technique may be a useful alternative in patients with known or unanticipated difficult tracheal intubations.

Competing interests None declared.

\section{References}

1. Henderson JJ, Popat MT, Latto IP, Pearce AC; Difficult Airway Society. Difficult Airway Society guidelines for management of the unanticipated difficult intubation. Anaesthesia 2004; 59: 675-94

2. Carron M, Freo U, Ori C. Bronchoscope-guided intubation through a Laryngeal Mask Airway Supreme in a patient with a difficult-to-manage airway. J Anesth 2009; 23: 613-5.

3. Joffe AM, Liew EC. Intubation through the LMA-Supreme ${ }^{\mathrm{TM}}$ : a pilot study of two techniques in a manikin. Anaesth Intensive Care 2010; 38: 33-8.

4. Matioc AA. Use of the Arndt airway exchanger catheter set with the Laryngeal Mask Airway Supreme. J Clin Anesth 2009; 21: 152-3.

5. Mathes AM, Wrobel $M$, Reus E, Rensing H, Grundmann $U$. Fiberoptic-guided intubation via the Laryngeal Mask Airway Supreme. J Clin Anesth 2008; 20: 322-3. 